\title{
Embracing Localization Inaccuracy with a Single Beacon
}

\author{
Anisur Rahman ${ }^{1}$ \\ Dept. of Computer Science and Engineering \\ East West University, Dhaka, Bangladesh
}

\author{
Vallipuram Muthukkumarasamy ${ }^{2}$ \\ School of Information and Communication Technology \\ Griffith University, Gold Coast, Australia
}

\begin{abstract}
This paper illustrates a new mechanism to determine the coordinates of the sensors using a beacon node and determines the definitive error associated with it. In UWSNs (underwater wireless sensor networks), actual and precise location of the deployed sensors which accumulate data is vital, because the accumulated data without the location information has less significance. Moreover it has limited value in the domain of location based services. In UWSN, trilateration or multilateration is exploited to assess the location of the deployed hosts; having three or more reference nodes to localize a deployed sensor is not pragmatic at all. On the other hand, nonlinear equations are usually solved in conventional method where degree-of-freedom is uncertain to lead to an exclusive solution. In this paper, associated localization inaccuracies has been shown for a unique configuration where a single beacon is used to determine the coordinates of three deployed sensors simultaneously. Cayley-Menger determinant is used for the configuration and system of nonlinear distance equations have been linearized for better accuracy and convergence. Simulations with Euclidean distances validate the propounded model and reflect the acquired accuracies in sensors' coordinates and bearings. Moreover, an experiment has been conducted with ultrasonic sensors in terrestrial environments to validate the proposed model; the associated inaccuracies were found to be generated from the distance measurement errors; on the other hand, considering Euclidean distances proves the model to be precise and accurate.
\end{abstract}

Keywords-Underwater localization; linearization; mobile beacon; Cayley-Menger determinant; bearing; underwater wireless sensor network

\section{INTRODUCTION}

Localization has become very prominent to provide location oriented services in terrestrial environment as well as in underwater. There are many underwater domains where localization of the submerged sensors and devices sometimes become crucial. Among them data collection for the sustenance of marine biome, finding lost objects, estuary monitoring and autonomous underwater vehicles control for military or research purposes are very common. These sorts of marine exploration are not only for the profusion of wealth the ocean has, it is also very vital for geological research to detect the movement of tectonic plates [1]. Some of these objectives require accurate localization for meaningful interpretation sensed data [2]. Erroneous localization is quite common; the degree of error in coordinates of the devices plays a vital role in comprehension of the problem and to provide solutions.
Location oriented services are become popular nowadays; to meet the demand, a plethora of methods have been proposed. These methods can be categorized as range-based and range-free schemes. Range based mainly depends on the distances between nodes, whereas range-free deals with profiling of the environment. Between these methods, accuracy of range based scheme is higher than that of range-free. In addition to that, former can be applied to a dynamic ever changing environment; whereas later performs well if environment is static. Generally multiple reference nodes are used for underwater localization; however, having one or many reference node is quite impractical. In this research paper, we have propounded a model that consists of single mobile beacon (boat/buoy) and at least three deployed sensors - a very realistic situation and typical configuration as depicted in Fig. 1. Moreover, acoustic signal is used for measuring inter node distances. In underwater wireless sensor networks, acoustic signals propagation and channels are naturally employed for distance measurements in contrast to radio signals $[1,3]$.

Having no preinstalled infrastructure in a dynamic configuration that can occur anytime is obvious. Hence, the proposed model kept in mind the natural occurrence and pragmatic nature of solutions. The model uses Cayley-Menger determinant and linearization of non-linear system of equations. Among few assumptions, surfing boat's plane and the deployed sensors' plane are in parallel state, which is sometimes the case in a water tank. Besides, voluntary and/or uncontrolled mobility of the nodes and in-situ measurement of speed of acoustic signals are kept for future exploration. This paper covers a simulation as well as hardware experiment in terrestrial environment. The results suggest negligible errors if true Euclidean distances are considered between beacon and deployed nodes. Positional errors found to be in $10^{-12}$ to $10^{-14} \mathrm{~m}$ range for a $150 \mathrm{~m}$ water column. If Gaussian error is added, the positional errors still remain within acceptable range for a sensor with a size of $0.25-0.5 \mathrm{~m}$ in length. It is conspicuous that distances between devices are the controlling factor for pin pointing the nodes. These negligible errors in simulations and experiments validate the proposed model.

The organization of the remaining paper is as follows. Section II focuses on acoustic signals propagation and distance measurement algorithm. Section III explains the proposed mathematical model; simulation, experimental results and analysis are elaborated in Sections IV, V and VI, respectively. Section VII states related works with associated constraints and finally conclusions in Section VIII. 


\section{Flight Time OF Acoustic Signals}

To determine the distances between beacon and deployed sensors, average speed of underwater acoustic signal is generally accepted to be $1500 \mathrm{~m} / \mathrm{s}$. Propagation of radio signals has limitations; acoustic signals travel much slower than radio signal; on the contrary, acoustic's propagation distance is much higher than that of radio in underwater. Nowadays, researchers shown a fervent interest in using radio underwater; in line of such a drive, we tend to use each of its merit. In hardware experiment in terrestrial, electrical signals has been used instead of radio; in the distance measurement method, we propose radio to be used for synchronization and acoustic for signal transfer.

\section{A. Assumptions:}

- Radio and acoustic signals can be generated simultaneously by the beacon node.

- Sensor nodes are stationary; beacon and sensor nodes are in parallel plane state.

Steps:

Radio and acoustic signals are generated simultaneous by beacon $S_{j}, j=4,5 \ldots$ at $t_{0}\left(S_{j}\right.$ : possible positions of the beacon)

Submerged sensors position $S_{i}, i=1,2,3$ as in Fig. 1

1) Sensors receive the radio signals immediately at $t_{R a(\text { rec })}=t_{0}+\varepsilon$ ( $\varepsilon$ : flight time of radio signal between beacon and sensors).

2) Sensor receives the acoustic signals after a while at $t_{A c(\text { rec })}$; here $\left(t_{A c(\text { rec })}-t_{0}\right)>>\left(t_{R a(\text { rec })}-t_{0}\right)$ due to high speed of radio signals.

Time of acoustic signals travelled from beacon to sensors:

$T_{i j(\text { Travel })}=t_{A c(\text { rec })}-t_{A c(\text { tra })}=t_{A c(\text { rec })}-t_{R a(t r a)} \because t_{A c(\text { tra })}=t_{R a(\text { tra })}$

$\therefore T_{i j(\text { Travel })} \approx t_{A c(\text { rec })}-t_{R a(\text { rec })} \quad \because t_{R a(\text { rec })}=t_{0}+\varepsilon \approx t_{R a(\text { tra })}$

$T_{i j(\text { Travel) }}$ : time beacon gets once sensors send with individual ID using radio signals

Eventually the distances between the beacon and sensors are computed by: $d_{i j}=\overline{\mathrm{v}} \times T_{i j(\text { travel) }}$ (here, $\overline{\mathrm{v}}$ is average speed of acoustic signals for the water column).

\section{COORDINATES DETERMINATION}

\section{B. Coordinates of the Sensors(Origing at the Sensor)}

The prime intention of localization process is to determine positions and bearing accurately with the use of gathered distances between beacon and deployed sensors. Distances are the only values available; traditionally it is assessed as a problem of optimization where objective functions that require minimization have residuals of distance equations. Typically in principle, number of equations should be at least equal to the number of unknown variables in the system. This concept known as degree-of-freedom may not guarantee a unique solution for a non-linear system of equations. Conventionally, multilateration is applied in solving this sort of non-linear system i.e. to determine locations or coordinates, in partial or full. In [4], Guevara et al. showed that initial condition is vital for the convergence of optimization algorithms; where they linearized the nonlinear equations and bypassed associated convergence problem.

Fig. 1 depicts the domain comprises of the beacon node $S_{j}, j=4,5 \ldots 9$ and deployed three sensors $S_{i}, i=1,2,3$. One of the underwater sensors $S_{i}, i=1,2,3$ is considered to be the origin $(0,0,0)$ of the Cartesian system and found trilateration equations are grouped in two. The distances between the beacon node and sensors are measured values $d_{14}, d_{24}, d_{34} \ldots$ and internode distances $d_{12}, d_{13}, d_{23}$ as well as volume of tetrahedron $V_{t}$ which is formed by surfaced beacon and underwater sensors are unknown. Depending on the local positioning system configuration depicted in Fig. 1, we determine the equations that include all known and unknown distances. So, Cayley-Menger determinant is used to determine the volume of tetrahedron $V_{t}$ as follows:

$288 V_{t}^{2}=\left|\begin{array}{ccccc}0 & 1 & 1 & 1 & 1 \\ 1 & 0 & d_{12}^{2} & d_{13}^{2} & d_{14}^{2} \\ 1 & d_{12}^{2} & 0 & d_{23}^{2} & d_{24}^{2} \\ 1 & d_{13}^{2} & d_{23}^{2} & 0 & d_{34}^{2} \\ 1 & d_{14}^{2} & d_{24}^{2} & d_{34}^{2} & 0\end{array}\right|$

By expanding (1), we obtain:

$$
\begin{aligned}
& d_{34}^{2} d_{23}^{2}-d_{34}^{2} d_{12}^{2}+d_{34}^{2} d_{13}^{2}-\frac{d_{14}^{2} d_{23}^{4}}{d_{12}^{2}}+d_{23}^{2} d_{14}^{2}+ \\
& \frac{d_{13}^{2} d_{14}^{2} d_{23}^{2}}{d_{12}^{2}}-\frac{d_{24}^{2} d_{13}^{4}}{d_{12}^{2}}+\frac{d_{13}^{2} d_{23}^{2} d_{24}^{2}}{d_{12}^{2}}+d_{13}^{2} d_{24}^{2}-d_{13}^{2} d_{23}^{2}- \\
& 144 \frac{V_{t}^{2}}{d_{12}^{2}}+\frac{d_{14}^{2} d_{23}^{2} d_{24}^{2}}{d_{12}^{2}}+\frac{d_{14}^{2} d_{23}^{2} d_{34}^{2}}{d_{12}^{2}}-\frac{d_{23}^{2} d_{24}^{2} d_{34}^{2}}{d_{12}^{2}}-\frac{d_{14}^{4} d_{23}^{2}}{d_{12}^{2}}+ \\
& \frac{d_{13}^{2} d_{24}^{2} d_{34}^{2}}{d_{12}^{2}}-\frac{d_{13}^{2} d_{14}^{2} d_{34}^{2}}{d_{12}^{2}}+\frac{d_{13}^{2} d_{14}^{2} d_{24}^{2}}{d_{12}^{2}}-\frac{d_{13}^{2} d_{24}^{4}}{d_{12}^{2}}-d_{34}^{4}+ \\
& d_{24}^{2} d_{34}^{2}+d_{14}^{2} d_{34}^{2}-d_{14}^{2} d_{24}^{2}=0
\end{aligned}
$$

By separating known variables from unknown, we get:

$$
\begin{aligned}
& d_{34}^{2}\left(d_{12}^{2}-d_{23}^{2}-d_{13}^{2}\right)+d_{14}^{2}\left(\frac{d_{23}^{4}}{d_{12}^{2}}-d_{23}^{2}-\frac{d_{13}^{2} d_{23}^{2}}{d_{12}^{2}}\right)+d_{24}^{2}\left(\frac{d_{13}^{4}}{d_{12}^{2}}-\frac{d_{13}^{2} d_{23}^{2}}{d_{12}^{2}}-d_{13}^{2}\right) \\
& -\left(d_{14}^{2} d_{24}^{2}+d_{14}^{2} d_{34}^{2}-d_{24}^{2} d_{34}^{2}-d_{14}^{4}\right) \frac{d_{23}^{2}}{d_{12}^{2}}-\left(d_{34}^{2} d_{24}^{2}-d_{14}^{2} d_{34}^{2}+d_{14}^{2} d_{24}^{2}-d_{24}^{4}\right) \\
& \frac{d_{13}^{2}}{d_{12}^{2}}+\left(144 \frac{V_{t}^{2}}{d_{12}^{2}}+d_{13}^{2} d_{23}^{2}\right)=\left(d_{24}^{2} d_{34}^{2}-d_{34}^{4}+d_{14}^{2} d_{34}^{2}-d_{14}^{2} d_{24}^{2}\right) \\
& \text { Here, } \quad\left(\frac{d_{23}^{4}}{d_{12}^{2}}-d_{23}^{2}-\frac{d_{13}^{2} d_{23}^{2}}{d_{12}^{2}}\right), \quad\left(\frac{d_{13}^{4}}{d_{12}^{2}}-\frac{d_{13}^{2} d_{23}^{2}}{d_{12}^{2}}-d_{13}^{2}\right), \\
& \left(d_{12}^{2}-d_{23}^{2}-d_{13}^{2}\right), \quad \frac{d_{23}^{2}}{d_{12}^{2}}, \quad \frac{d_{13}^{2}}{d_{12}^{2}}, \quad \text { and }\left(144 \frac{V_{t}^{2}}{d_{12}^{2}}+d_{13}^{2} d_{23}^{2}\right) \text { are }
\end{aligned}
$$

considered as unknown variables. 


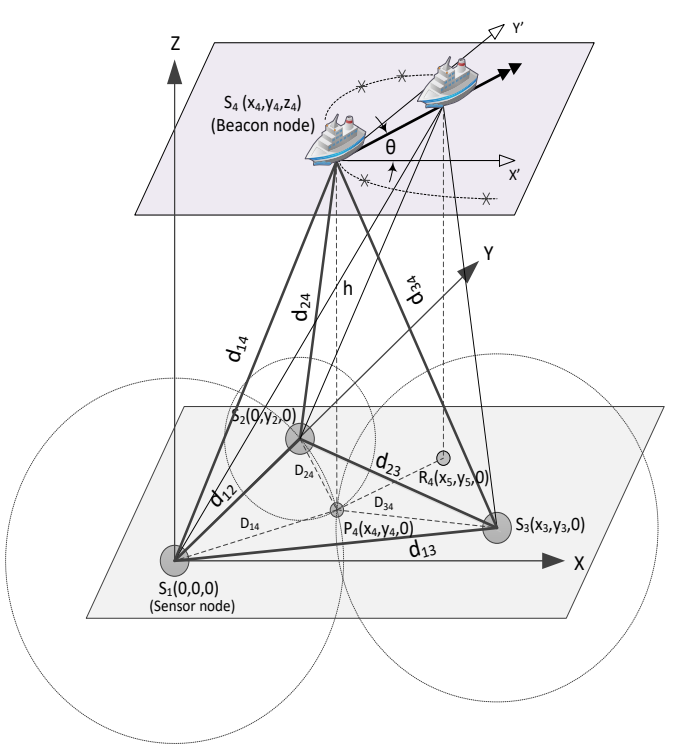

Fig. 1. Coordinates Determination.

So, we get:

$$
\begin{aligned}
& d_{14}^{2} X_{1}+d_{24}^{2} X_{2}+d_{34}^{2} X_{3}-\left(d_{14}^{2}-d_{34}^{2}\right)\left(d_{24}^{2}-d_{14}^{2}\right) X_{4} \\
& -\left(d_{24}^{2}-d_{14}^{2}\right)\left(d_{34}^{2}-d_{24}^{2}\right) X_{5}+X_{6}=\left(d_{24}^{2}-d_{34}^{2}\right)\left(d_{34}^{2}-d_{14}^{2}\right)
\end{aligned}
$$

Equation (2) has six unknowns, which matches with the linear form of $a_{1} x_{1}+a_{2} x_{2}+\cdots+a_{n} x_{n}=b_{1}$. As there are six unknowns, to solve we have taken six measurements. The measurements can be taken following aforesaid procedure by steering the beacon node $S_{j}, j=4,5 \ldots .9$ to different area of the water surface. These six measurements can be taken at the vicinity of $S_{4}$, it is worth mentioning that the deployed underwater sensors are considered to be static at the time of measuring distances. By omitting references to the variables we get an array of all coefficients recognized as augmented matrix, where first row of the array represents the first linear equation and so on as $A X=b$ form. So, system of linear equations can be expressed as following:

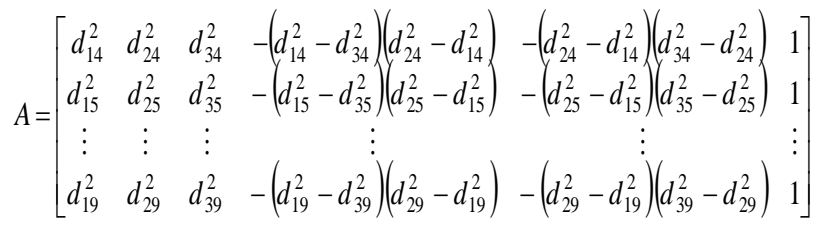

$$
X=\left[\begin{array}{c}
\left(\frac{d_{23}^{4}}{d_{12}^{2}}-d_{23}^{2}-\frac{d_{13}^{2} d_{23}^{2}}{d_{12}^{2}}\right) \\
\left(\frac{d_{13}^{4}}{d_{12}^{2}}-\frac{d_{13}^{2} d_{23}^{2}}{d_{12}^{2}}-d_{13}^{2}\right) \\
\left(d_{12}^{2}-d_{23}^{2}-d_{13}^{2}\right) \\
\frac{d_{23}^{2}}{d_{12}^{2}} \\
\frac{d_{13}^{2}}{d_{12}^{2}} \\
\left(144 \frac{V_{t}^{2}}{d_{12}^{2}}+d_{13}^{2} d_{23}^{2}\right)
\end{array}\right] \quad b=\left[\begin{array}{c}
\left(d_{24}^{2}-d_{34}^{2}\right)\left(d_{34}^{2}-d_{14}^{2}\right) \\
\left(d_{25}^{2}-d_{35}^{2}\right)\left(d_{35}^{2}-d_{15}^{2}\right) \\
\vdots \\
\left(d_{29}^{2}-d_{39}^{2}\right)\left(d_{29}^{2}-d_{19}^{2}\right)
\end{array}\right]
$$

From the above representation, after finding $X_{1}, X_{2}, X_{3}$, $X_{4}, X_{5}$ and $X_{6}$ we calculate $d_{12}, d_{13}$ and $d_{23}$ as follows:

$$
d_{12}^{2}=\frac{X_{3}}{\left(1-X_{4}-X_{5}\right)}, d_{13}^{2}=\frac{X_{3} X_{5}}{\left(1-X_{4}-X_{5}\right)}, d_{23}^{2}=\frac{X_{3} X_{4}}{\left(1-X_{4}-X_{5}\right)}
$$

Here, coordinates of deployed underwater sensors $S_{1}, S_{2}$ and $S_{3}$ are considered to be $(0,0,0),\left(0, y_{2}, 0\right)$ and $\left(x_{3}, y_{3}, 0\right)$ respectively; so, the inter sensor distances can be stated with respect to coordinates of the sensors are as follows:

$d_{12}^{2}=y_{2}^{2}, d_{13}^{2}=x_{3}^{2}+y_{3}^{2}, d_{23}^{2}=x_{3}^{2}+\left(y_{3}-y_{2}\right)^{2}$

So, the unknown variables can be derived as:

$$
y_{2}=d_{12}, \quad y_{3}=\frac{d_{12}^{2}+d_{13}^{2}-d_{23}^{2}}{2 d_{12}}, x_{3}=\sqrt{\left(d_{13}^{2}-\left(\frac{d_{12}^{2}+d_{13}^{2}-d_{23}^{2}}{2 d_{12}}\right)^{2}\right)}
$$

Here $d_{12}, d_{13}$ and $d_{23}$ are computed distances. Coordinates of the sensors for the proposed problem domain are illustrated in Table I as follows.

\section{Coordinates of the Sensors (origing at the beacon)}

So far we calculated the coordinates of the sensors with respect to $S_{1}$ which was considered to be the origin of the system. To find with respect to beacon, following steps need to be followed.

By incorporating depth sensor with the deployed nodes, the depth $h$ in Fig. 1 can be measured as depicted in [5]. After measuring the vertical distance $h$ in between the beacon node $S_{4}\left(x_{4}, y_{4}, z_{4}\right)$ and the $X Y$ plane, we can assume the projected coordinate of the beacon node $S_{4}\left(x_{4}, y_{4}, z_{4}\right)$ on the plane $X Y$ is $P_{4}\left(x_{4}, y_{4}, 0\right)$. To find $x_{4}$ and $y_{4}$, we can apply trilateration in the following manner assuming the distances between $S_{1}, S_{2}, S_{3}$ and $P_{4}$ are $D_{14}, D_{24}$ and $D_{34}$ respectively and device following relations.

$$
\begin{aligned}
& D_{14}^{2}=x_{4}^{2}+y_{4}^{2} \\
& D_{24}^{2}=x_{4}^{2}+\left(y_{4}-y_{2}\right)^{2} \\
& D_{34}^{2}=\left(x_{4}-x_{3}\right)^{2}+\left(y_{4}-y_{3}\right)^{2}
\end{aligned}
$$

TABLE. I. COORDINATES OF THE SENSORS

\begin{tabular}{|l|l|}
\hline Sensors & Coordinates \\
\hline$S_{1}$ & $(0,0,0)$ \\
\hline$S_{2}$ & $\left(0, d_{12}, 0\right)$ \\
\hline$S_{3}$ & $\left(\sqrt{\left(d_{13}^{2}-\left(\frac{d_{12}^{2}+d_{13}^{2}-d_{23}^{2}}{2 d_{12}}\right)^{2}\right)}, \frac{d_{12}^{2}+d_{13}^{2}-d_{23}^{2}}{2 d_{12}}, 0\right)$ \\
\hline
\end{tabular}


From (3), (4) and (5) we obtain the projected beacon's coordinates $P_{4}\left(x_{4}, y_{4}, 0\right)$, where

$$
\begin{aligned}
& x_{4}=\frac{1}{2 d_{12}} \sqrt{\left(4 d_{12}^{2} D_{14}^{2}-\left(D_{14}^{2}-D_{24}^{2}+d_{12}^{2}\right)^{2}\right.}, \\
& y_{4}=\frac{1}{2 d_{12}}\left(D_{14}^{2}-D_{24}^{2}+d_{12}^{2}\right)
\end{aligned}
$$

As $d_{14}, d_{24}$ and $d_{34}$ are the hypotenuse of the $\Delta S_{1} P_{4} S_{4}$, $\Delta S_{2} P_{4} S_{4}$ and $\Delta S_{3} P_{4} S_{4}$ respectively, so it is possible to obtain $D_{14}, D_{24}$ and $D_{34}$ using Pythagorean Theorem. So the coordinate of the beacon node $S_{4}\left(x_{4}, y_{4}, z_{4}\right)$ would be ${ }^{\left(x_{4}, y_{4}, h\right)}$ where all the elements are known.

$$
\begin{aligned}
& \therefore S_{4}\left(x_{4}, y_{4}, h\right)= \\
& S_{4}\left(\frac{1}{2 d_{12}} \sqrt{\left(4 d_{12}^{2} D_{14}^{2}-\left(D_{14}^{2}-D_{24}^{2}+d_{12}^{2}\right)^{2}\right.}, \frac{1}{2 d_{12}}\left(D_{14}^{2}-D_{24}^{2}+d_{12}^{2}\right), h\right)
\end{aligned}
$$

By linear transformation, the origin (one of the sensor's coordinates) of the Cartesian system has been transferred to coordinates of the beacon; found sensors' coordinates with respect to beacon $S_{4}$ are depicted as in Table II.

TABLE. II. COORDINATES WITH RESPECT TO THE BEACON

\begin{tabular}{|l|l|}
\hline & Coordinates \\
\hline$S_{4}$ & $(0,0,0)$ \\
\hline$S_{1}$ & $\left(\frac{\sqrt{4 d_{12}^{2} D_{14}^{2}-\left(D_{14}^{2}-D_{24}^{2}+d_{12}^{2}\right)^{2}}}{2 d_{12}},-\frac{1}{2 d_{12}}\left(D_{14}^{2}-D_{24}^{2}+d_{12}^{2}\right),-h\right)$ \\
\hline$S_{2}$ & $\left(\frac{\sqrt{4 d_{12}^{2} D_{14}^{2}-\left(D_{14}^{2}-D_{24}^{2}+d_{12}^{2}\right)^{2}}}{2 d_{12}}, \frac{1}{2 d_{12}}\left(d_{12}^{2}-D_{14}^{2}+D_{24}^{2}\right),-h\right)$ \\
\hline$S_{3}$ & $\left(\begin{array}{l}\left(\sqrt{\left(d_{13}^{2}-\left(\frac{d_{12}^{2}+d_{13}^{2}-d_{23}^{2}}{2 d_{12}}\right)^{2}\right)}-\frac{\sqrt{4 d_{12}^{2} D_{14}^{2}-\left(D_{14}^{2}-D_{24}^{2}+d_{12}^{2}\right)^{2}}}{2 d_{12}}\right), \\
2 d_{12}\left(d_{13}^{2}-d_{23}^{2}-D_{14}^{2}+D_{24}^{2}\right),\end{array}\right)$ \\
\hline
\end{tabular}

\section{SIMULATION RESULTS}

A simulation environment is created in Matlab for the aforesaid problem domain taking a sensor as the beacon (boat/buoy) and three sensors. The depth of the problem domain is considered for a $150 \mathrm{~m}$ water column. The complexity of the simulated environment is discussed as well as positional errors for sensors have been elaborated in Table III. In [6], coordinates of the sensors with respect to beacon has been shown. With the simulated environment, it is possible to determine 3D coordinates of the sensors with bearing information; as the beacon is at the surface of the water, the coordinates could be known by GPS.

To validate the proposed model, three sensors are placed in random fashion on the XY plane and beacon, which is mobile is kept above $\mathrm{XY}$ plane. As the positions are random, one of the sensors is considered as the origin of the Cartesian system; the other sensors are on the $\mathrm{y}$-axis and on any point of $\mathrm{XY}$ plane respectively. The model suggests that the beacon should be steered in six different locations to measure distances between the sensor above and the sensors on the XY plane. However, the sensors on the XY plane are considered to be static while the sensor (beacon) steers in six different locations. To verify the proposed mathematical model, at first true Euclidean distances were considered; afterward Gaussian errors in the distances added.

The orientations of the mobile beacon and its effects have been explored in [7]; straight line, circular line and angular Archimedean spirals of different radius $(5-50 \mathrm{~m})$ are among them. Archimedean arc lengths of spirals of different radius are calculated according to (6) and (7).

$r=a+b \theta$

$L=\int_{a}^{b} \sqrt{r^{2}+\left(\frac{d r}{d \theta}\right)^{2}} d \theta$

Here, $r$ and $\theta$ are distance from origin and span from $a$ to $b$ respectfully.

We have found that true Euclidean distances between beacon and deployed sensors produce negligible errors. For a $150 \mathrm{~m}$ water column, positional errors remain within $10^{-12}$ to $10^{-14} \mathrm{~m}$ range, so we can conclude that for a sensor that has radius in meters, this negligible error validates the proposed mathematical model. The accuracy of the coordinates are denoted in Table III. It is worth noting that these errors in nanometre range produced from the linearization process of non-linear equations. It also illustrates that the beacon's orientation can be in any form other than straight movement; straight line mobility generates singular matrix. Moreover, span of the sensor mobile trajectory has no effect on coordinates. However, if the distance measurements are taken in close proximity, then generated errors can be kept minimal.

TABLE. III. POSITIONAL ERRORS FOR $\mathrm{S}_{1}, \mathrm{~S}_{2}$ AND $\mathrm{S}_{3}$ (WITH RESPECT TO BEACON :EUCLIDEAN DISTANCES)

\begin{tabular}{|l|l|l|l|}
\hline \multirow{2}{*}{$\begin{array}{l}\text { Orientation } \\
\text { (circular) } \\
\text { (radius) }\end{array}$} & \multicolumn{3}{|l|}{ Positional Error } \\
\cline { 2 - 4 } & $\mathrm{S}_{1}(\mathrm{~m})$ & $\mathrm{S}_{2}(\mathrm{~m})$ & $\mathrm{S}_{3}(\mathrm{~m})$ \\
\hline $5 \mathrm{~m}$ & $1.11 \times 10^{-12}$ & $1.99 \times 10^{-12}$ & $3.51 \times 10^{-12}$ \\
\hline $10 \mathrm{~m}$ & $4.39 \times 10^{-13}$ & $8.93 \times 10^{-13}$ & $1.59 \times 10^{-12}$ \\
\hline $15 \mathrm{~m}$ & $1.44 \times 10^{-13}$ & $2.15 \times 10^{-13}$ & $1.15 \times 10^{-12}$ \\
\hline $20 \mathrm{~m}$ & $7.12 \times 10^{-15}$ & $5.70 \times 10^{-14}$ & $7.31 \times 10^{-13}$ \\
\hline $50 \mathrm{~m}$ & $7.09 \times 10^{-15}$ & $4.24 \times 10^{-14}$ & $1.05 \times 10^{-13}$ \\
\hline $\begin{array}{l}\text { Orientation } \\
\text { (spiral) } \\
\text { (single turn } \\
\text { increase) }\end{array}$ & Positional Error & \multicolumn{2}{|l}{} \\
\hline $5 \mathrm{~m}$ & $\mathrm{~S}_{1}(\mathrm{~m})$ & $\mathrm{S}_{2}(\mathrm{~m})$ & $\mathrm{S}_{3}(\mathrm{~m})$ \\
\hline $10 \mathrm{~m}$ & $6.05 \times 10^{-13}$ & $1.20 \times 10^{-12}$ & $3.24 \times 10^{-12}$ \\
\hline $15 \mathrm{~m}$ & $4.56 \times 10^{-13}$ & $9.26 \times 10^{-13}$ & $2.61 \times 10^{-12}$ \\
\hline $20 \mathrm{~m}$ & $8.54 \times 10^{-13}$ & $1.67 \times 10^{-12}$ & $3.15 \times 10^{-12}$ \\
\hline $50 \mathrm{~m}$ & $5.75 \times 10^{-13}$ & $1.15 \times 10^{-12}$ & $7.75 \times 10^{-13}$ \\
\hline
\end{tabular}




\section{EXPERIMENTAL SETUP AND RESULTS}

\section{A. Description of the Setup}

The problem domain suggests that at least three sensors need to be deployed underwater and a single beacon would remain on the surface of the water. Usually sensors are deployed in various numbers underwater to accumulate data; the number can range from a few to many. Among various methods, in our experiment flight time of the signals is considered to measure distances between beacon and deployed sensors as in [8]. We also assume that the experimental water column is considered to be homogeneous; though temperature, salinity and pressure (depth) affects the speed of acoustic signals. Most of the papers consider $1500 \mathrm{~m} / \mathrm{s}$ to be the speed of acoustic signals underwater; however, speed measurement of acoustic signal is not covered in this paper. Besides, signals propagation in this adverse environment and various other factors left unaddressed.

The experiment requires us to take multiple measurements according to the proposed model; we have also considered the plane i.e. the plane where beacon surfs and the plane of sensors to be in parallel state. However, non-parallel state scenario has not been considered in this paper. Besides, for simplicity, we considered the deployed sensors would be in static state while taking six measurements. The mobility of the sensors we tend to address in future. Fig. 1 illustrates a solvable configuration of the experiment in line of proposed model.

\section{B. Devices and Setup}

The experiment has been performed with an Arduino board connected to four HC-SR04 ultrasonic sonic sensors as in Fig. 2 and 3. Ultrasonic sensors are mainly used to determine distances with bouncing technique; which is, generated signal bounces back from the nearest obstacles that are positioned in front of the sensor. If the object's position is placed in angular fashion, i.e., is positioned not right in front of the sensor, HCSR04 sensor will not be able to receive the bounced back signals. In this experiment we have customized the sensors as such that it does not measure the distance with bouncing signal,

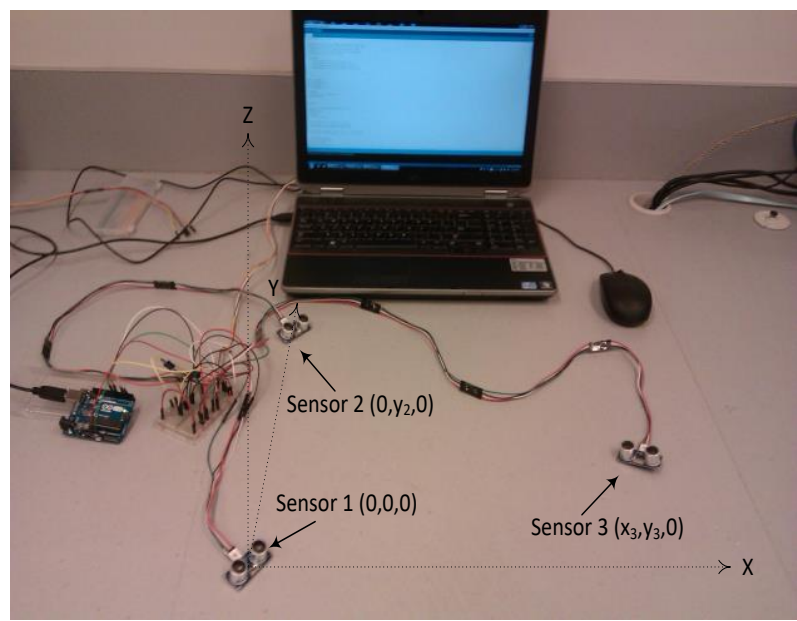

Fig. 2. Experimental Setup with Sensors in Terrestrial Environment with Sensors on the Floor and Beacon at the Ceiling.

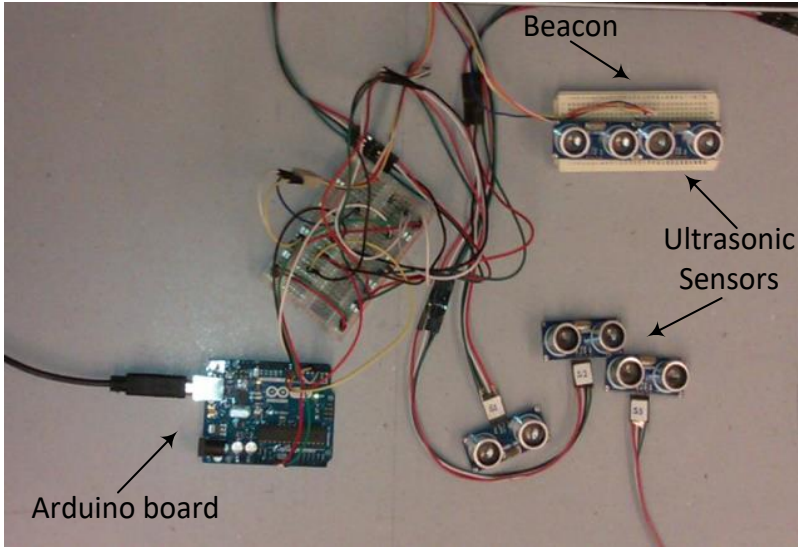

Fig. 3. Different ultrasonic Sensors and Board used in Experiment.

whereas the table top sensors detect the presence of signal that has been generated by the beacon sensor at the ceiling.

\section{Experimental Results}

The experiment has been conducted in two different orientations; four tests have been performed in each scenario as in Fig. 4 and 5. For each scenario positional errors are shown in Tables IV and V.

For the scenario 1, we can see that keeping the origin at the $S_{1}$, positional error for $S_{2}$ and $S_{3}$ are within 0.2 to $4 \mathrm{~cm}$ range. Accuracy in distance measurements with the ultrasonic sensor generates accurate positional error: $0.17 \mathrm{~cm}$, whereas in extreme case it is $3.85 \mathrm{~cm}$.

For the scenario 2, we can see that keeping the origin at the $S_{1}$, positional error for $S_{2}$ and $S_{3}$ are within 0.5 to $6 \mathrm{~cm}$ range. Accuracy in distance measurements with the ultrasonic sensor generates accurate positional error: $0.47 \mathrm{~cm}$, whereas in extreme case it is $5.90 \mathrm{~cm}$.

TABLE. IV. POSITIONAL ERROR FOR SCENARIO 1

\begin{tabular}{|l|l|l|l|}
\hline & $\mathrm{S}_{1}(\mathrm{~cm})$ & $\mathrm{S}_{2}(\mathrm{~cm})$ & $\mathrm{S}_{3}(\mathrm{~cm})$ \\
\hline Test 1 & 0 & 0.17 & 0.42 \\
\hline Test 2 & 0 & 0.55 & 0.98 \\
\hline Test 3 & 0 & 0.73 & 1.02 \\
\hline Test 4 & 0 & 2.28 & 3.85 \\
\hline & & & \\
\hline
\end{tabular}

TABLE. V. POSITIONAL ERROR FOR SCENARIO 2

\begin{tabular}{|l|l|l|l|}
\hline & $\mathrm{S}_{1}(\mathrm{~cm})$ & $\mathrm{S}_{2}(\mathrm{~cm})$ & $\mathrm{S}_{3}(\mathrm{~cm})$ \\
\hline Test 1 & 0 & 1.28 & 3.41 \\
\hline Test 2 & 0 & 1.12 & 2.97 \\
\hline Test 3 & 0 & 0.47 & 2.71 \\
\hline Test 4 & 0 & 2.11 & 5.90 \\
\hline
\end{tabular}


Scenario 1:

Original coordinates of sensors:

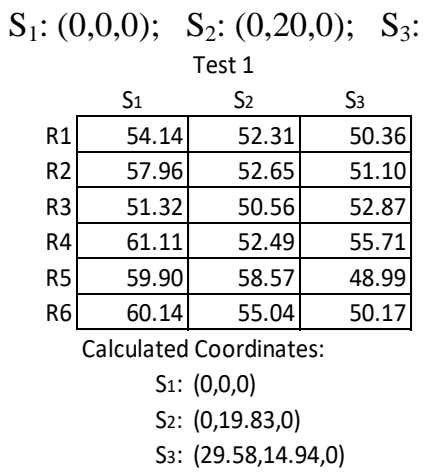

\begin{tabular}{|c|c|c|c|}
\hline \multicolumn{4}{|c|}{ Test 2} \\
\hline & $S_{1}$ & $\mathrm{~S}_{2}$ & $\mathrm{~S}_{3}$ \\
\hline R1 & 62.57 & 57.27 & 52.20 \\
\hline R2 & 60.31 & 54.78 & 53.17 \\
\hline R3 & 53.39 & 52.60 & 55.01 \\
\hline R4 & 63.58 & 54.62 & 57.96 \\
\hline R5 & 62.32 & 60.94 & 50.98 \\
\hline $\mathrm{R} 6$ & 56.34 & 54.42 & 52.40 \\
\hline & ulated & rdinat & \\
\hline & S1: & & \\
\hline & S2: & $20.55,0)$ & \\
\hline & S3: & $.88,15$ & \\
\hline
\end{tabular}

\begin{tabular}{|c|c|c|c|}
\hline \multicolumn{4}{|c|}{ Test 3} \\
\hline & $\mathrm{S}_{1}$ & $\mathrm{~S}_{2}$ & $\mathrm{~S}_{3}$ \\
\hline R1 [ & 60.76 & 55.20 & 53.57 \\
\hline R2 & 56.76 & 54.83 & 52.79 \\
\hline R3 & 53.80 & 53.00 & 55.43 \\
\hline R4 & 64.06 & 55.03 & 58.40 \\
\hline R5 & 62.79 & 61.41 & 51.36 \\
\hline R6 & 63.04 & 57.71 & 52.60 \\
\hline & ulatec & rdinate & \\
\hline & S1: & & \\
\hline & S2: & $20.73,0)$ & \\
\hline & S3: & $.93,15.4$ & \\
\hline
\end{tabular}

\begin{tabular}{|c|c|c|c|}
\hline \multicolumn{4}{|c|}{ Test 4} \\
\hline & $\mathrm{S}_{1}$ & $\mathrm{~S}_{2}$ & $\mathrm{~S}_{3}$ \\
\hline R1 & 65.58 & 59.57 & 57.81 \\
\hline R2 & 61.26 & 59.18 & 56.97 \\
\hline R3 & 58.06 & 57.20 & 59.82 \\
\hline R4 & 69.13 & 59.39 & 63.03 \\
\hline R5 & 67.77 & 66.27 & 55.43 \\
\hline R6 & 68.04 & 62.28 & 56.76 \\
\hline & alculated & ordinate & \\
\hline & $\mathrm{S}_{1}:$ & & \\
\hline & S2: & $22.28,0)$ & \\
\hline & S3: & 4816 & \\
\hline
\end{tabular}

Fig. 4. Calculated Coordinates of the Sensors According to Scenario 1.

Scenario 2:

Original coordinates of sensors:

$$
\mathrm{S}_{1}:(0,0,0) ; \quad \mathrm{S}_{2}:(0,25,0) ; \mathrm{S}_{3}:(35,10,0)
$$
Test 1

\begin{tabular}{l|r|r|r|}
\multicolumn{1}{c}{} & \multicolumn{1}{c}{ S1 } & \multicolumn{1}{c}{ S2 } \\
\cline { 2 - 4 } R1 & 59.85 & 57.26 & 48.71 \\
\cline { 2 - 4 } R2 & 50.32 & 53.25 & 51.74 \\
R3 & 53.70 & 53.04 & 49.86 \\
\cline { 2 - 4 } R4 & 51.20 & 49.57 & 53.90 \\
R5 & 57.65 & 55.37 & 48.92 \\
\cline { 2 - 4 } R6 & 55.25 & 50.62 & 52.43 \\
\cline { 2 - 3 } & \multicolumn{3}{c}{ Calculated Coordinates: }
\end{tabular}

$$
\text { S1: }(0,0,0)
$$$$
\text { S2: }(0,23.72,0)
$$$$
\text { S3: }(33.26,12.94,0)
$$

\begin{tabular}{l|l|l|l|}
\multicolumn{4}{c}{ Test 2 } \\
\multicolumn{1}{c}{} & \multicolumn{1}{c}{ S1 } & \multicolumn{1}{c}{ S3 } \\
\cline { 2 - 4 } R1 & 65.34 & 62.51 & 53.19 \\
\hline R2 & 54.94 & 58.15 & 56.49 \\
\hline R3 & 55.90 & 54.13 & 58.85 \\
\cline { 2 - 4 } R4 & 58.63 & 57.91 & 54.44 \\
\cline { 2 - 4 } R5 & 62.95 & 60.46 & 53.42 \\
\cline { 2 - 4 } R6 & 60.32 & 55.26 & 57.25 \\
\cline { 2 - 4 } & & &
\end{tabular}

Test 3

\begin{tabular}{|c|c|c|c|}
\hline \multicolumn{4}{|c|}{ Test 4} \\
\hline & $\mathrm{S}_{1}$ & $\mathrm{~S}_{2}$ & $\mathrm{~S}_{3}$ \\
\hline R1 & 65.85 & 63.24 & 55.88 \\
\hline R2 & 57.47 & 60.83 & 59.09 \\
\hline R3 & 61.33 & 60.58 & 56.95 \\
\hline R4 & 68.35 & 65.40 & 55.64 \\
\hline R5 & 58.47 & 56.62 & 61.57 \\
\hline R6 & 63.10 & 57.81 & 59.89 \\
\hline
\end{tabular}

\begin{tabular}{l|l|l|l|}
\multicolumn{4}{c}{ Test 3 } \\
\multicolumn{1}{c}{} & \multicolumn{1}{c}{ S1 } & \multicolumn{1}{c}{ S2 } \\
\cline { 2 - 4 } R1 & 56.77 & 52.01 & 53.88 \\
\cline { 2 - 4 } R2 & 51.71 & 54.73 & 53.17 \\
R3 & 55.18 & 54.51 & 51.24 \\
\cline { 2 - 4 } R4 & 52.61 & 50.94 & 55.39 \\
\cline { 2 - 4 } R5 & 59.25 & 56.90 & 50.28 \\
\cline { 2 - 4 } R6 & 61.50 & 58.84 & 50.06 \\
\cline { 2 - 4 } & \multicolumn{3}{|c}{}
\end{tabular}

$$
\begin{aligned}
& \text { S1: }(0,0,0) \\
& \text { S2: }(0,24.53,0) \\
& \text { S3: }(34.33,12.63,0)
\end{aligned}
$$

$$
\begin{aligned}
& \text { S1: }(0,0,0) \\
& \text { S2: }(0,27.11,0) \\
& \text { S3: }(37.85,15 \cdot 17,0)
\end{aligned}
$$

S1: $(0,0,0)$

S2: $(0,26.12,0)$

S3: $(36.55,12.54,0)$

Fig. 5. Calculated Coordinates of the Sensors According to Scenario 2.

\section{ANALYSIS AND DISCUSSIONS}

Pragmatic approach of this method is having a single beacon at the water surface and sensors underwater. Having a boat or buoy in the time of crisis is natural than having multiples. Besides, this method won't require any preinstalled reference points; but still it can determine the coordinates of the sensors dynamically with negligible error. The proposed model is validated by considering true Euclidean distances; later on Gaussian error has been added to acquired distances. By taking true Euclidean distances, it has been shown that accuracy of distance determination would generate accuracy in coordinates.

The acquired result has been reinforced by experimenting three ultrasonic sensors on the table top and a beacon above those emulating the configuration as depicted earlier. Flight time i.e. the propagation time of acoustic signals is used to calculate the distances between beacon and underneath sensors. This way of measuring distance helps us to avoid multipath fading affect present in RSSI. It also shows that it is possible to calculate the distances between sensors as the pulses travel the shortest Euclidean distance. Fig. 6 shows the intended distance to be measured and acquired distances are considered to calculate coordinates of the sensors in Matlab.

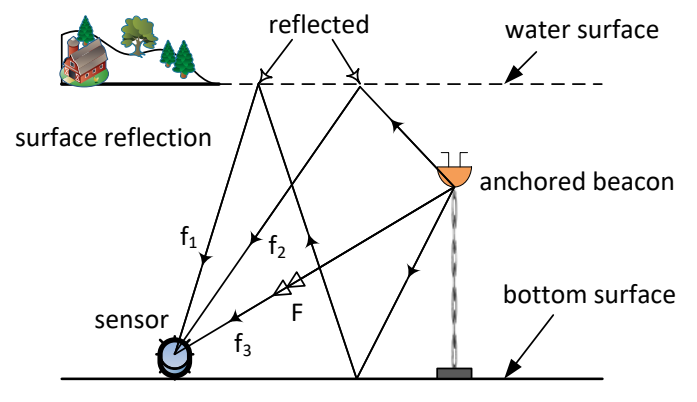

Fig. 6. Multipath Fading can be Avoided to Measure Shortest Euclidean Distance.

\section{A. Internode Distances from Acoustic Signals' Flight Time}

Ultrasonic sensor at the top generates TTL impulses in 40 $\mathrm{KHz}$ frequency and 3 sensors at the bottom receive the pulses and record the time. Speed of acoustic signal in normal environment in terrestrial is considered $340 \mathrm{~m} / \mathrm{s}$ i.e. it takes $29 \mu$ s to travel $1 \mathrm{~cm}$ of distance; by measuring the flight time of the acoustic signal it then calculates the distance between the beacon and the sensors. It is worth noting that clocks of all the sensors including the beacon at the top are synchronized as it is connected to the same Arduino board; besides the pulse generation time and a sample of generated impulse by the beacon is made available to the sensors to determine the signals' arrival time as soon as it travel the shortest Euclidean distance.

\section{B. Problems and Challenges Encountered in Experiments}

Mathematical model has been validated in simulated environment concluding the accuracy of the distance measurements are the limiting factor of the precise coordinates determination. Different scenario has been chosen for the experiment and each scenario is tested multiple times. In each 
test we needed to read the distances six times from different positions of the beacon. While reading the values time to time some readings were such that it does not comply with the real distance, and eventually did not converge in Matlab. In those cases we had to go for another test that ultimately converges. In each reading the measured distances for $S_{1}, S_{2}$ and $S_{3}$ have to be consistent.

The off the shelf sensors has $12^{0}$ sentry angle which limits the beacon's movement span. Within this sentry angle, acquired positional errors are in the range of $0.5-6 \mathrm{~cm}$. Having an acoustic sensor with $4.5 \times 1.5 \times 1.5 \mathrm{~cm}$ in size, this range of error is within acceptable range. In simulation, Cartesian coordinates has been used and one of the sensor is placed at the origin. The $150 \mathrm{~m}$ water column has not been emulated in Matlab as distances between beacon and sensors are determined by considering flight time and its average speed. Besides, multipaths fading due to obstruction are left unaddressed in this paper. The simulation results also suggest that beacon's movement has limited effect on coordinates of the sensor. We have shown that angular and circular movement do not affect localization method except moving the beacon in straight line which leads to unsolvable singular matrix. In practice, moving in a very straight line is rare where current is present. In the method, expanded Cayley-Menger determinant is used calculate the volume of tetrahedron and derived equations are non-linear. Besides, due to six unknown variables, six individual measurements were necessary to solve linearized system of equations.

\section{RELATED WORKS}

There are many localization algorithms where known values of few sensors are usually used to determine their positions, among them distances between sensor in time interval and bearing of any moment are common. Localization in terrestrial is popular field of research due to the demand of location oriented services. However, techniques that are used in terrestrial sometime may not be used in UWSNs due to different characteristics and heterogeneous nature of underwater world. Guevara et al. proposed a closed-form method where positions of nodes are not required to localize multiple static reference nodes in [4]; distances between mobile nodes and static reference nodes are sufficient for the proposed method. Recently, the preciseness of the model with Euclidean distances between beacon and sensors has been elaborated in [6]. The paper showed that the produced negligible errors were due to linearization process of the method. Moreover, the effect of Gaussian noise in distance measurements and its implication on coordinates and bearing have been shown in [7].

Signals propagation model for this type of heterogeneous environment is quite different than that of terrestrial environment; Chandrasekhar et al. explored and showed signal propagation difficulties and limitations in UWSNs in [9]. Among the plethora of proposed localization algorithms, few have shown the merit to be explored. In [10], three dimensional Euclidean distance estimation has been proposed; where a specific number of submerged nodes are required to measure internode distances. Besides, generated error in distance measurement propagates through the system due to its recursive behaviour. On the other hand, proposed method in [11] requires mobile sensors to communicate with moored nodes (buoys) for detecting its location. Besides, 3D positioning system in [12] requires four separate positions to determine the coordinates of the beacon. The major limitation of these methods are static inherently, dynamicity of localization is absent. Node requires to be moored to the waterbed in advance, which is cumbersome as well as possess inconveniences.

Duff and Muller delineates incorporates nonlinear least square method in their model to solve the system of multilateration equations in [13]. In the proposed model, authors have focused on the necessity of multiple equations that is gathered from different locations of the nodes - a criterion of degree-of-freedom analysis of system of linear equations. The proposed model has been validated in [14] by incorporating of Kalman filter with many folds. However, the complexity and inherent nature of degree-of-freedom have been shown in [15], which showed that it does not guarantee a unique solution for nonlinear system, such as trilateration. Trilateration or multilateration may not be solved when only parameter available is distances between nodes. Moreover, the prerequisites to have initial configuration of nodes have been justified with rigidity theory as well.

This paper focuses on the method of determining coordinates and bearing of submerged sensors and associated inaccuracies; a pragmatic method has been proposed where a single mobile node will be used to localize deployed submerged sensors. Having a single beacon without any preinstalled reference points is dynamic by nature. Recently, localization of submerged nodes with a single beacon has been illustrated in [16] for non-parallel state situation, i.e. the plane where the beacon surfs and the plane where three of the submerged sensors are deployed are not parallel. The paper also showed that it is the distance between beacon and nodes which affects the model, not the state of the planes.

\section{CONCLUSIONS}

Steadfast positioning is indispensable in various applications and services due to safety and research. Location oriented services are the demand of time regardless of terrestrial or underwater environment for conveniences. Among the plethora of localization models, this paper illustrates associated inaccuracies in the proposed mathematical model to determine coordinates and bearings of sensors with a single beacon (node) in real time considering the environment. The practical orientation of the proposed domain and the aforesaid model has been validated with simulation results having negligible errors. This paper showed the practicality of having a single boat/buoy (beacon) at the water surface while localizing deployed nodes instead of three or more beacons.

Simulation results showed negligible errors in coordinates of the nodes while Euclidean distances were considered. It also delineates the produces errors are due to erroneous distance determination between beacon and nodes. The accuracy of the distance measurements lead to pin pointing the nodes. Moreover, beacon's mobility and span has limited or almost no effect on coordinates. Experimental results performed in terrestrial environment also suggest that the inter node 
distances can be measured by calculating the flight time of acoustic signals with the help of electrical signal for clock synchronization. As acoustic signal is affected by environmental constraints; inaccuracies in distance determinations were due to signals propagations and signal processing limitation of the ultrasonic sensors. Eventually coordinates of the sensors can be determined with a single beacon following proposed model with inevitable error due to inaccuracies in distance determinations.

In future, we intend to address voluntary mobility of the autonomous underwater vehicles and involuntary mobility of the freely deployed sensors due to currents in the proposed model.

\section{REFERENCES}

[1] J. H. Cui, J. Kong, M. Gerla, and S. Zhou, "The challenges of building mobile underwater wireless networks for aquatic applications," Network, IEEE, vol. 20, pp. 12-18, 2006.

[2] L. Hu, B. Liu, K. Zhao, X. Meng, and F. Wang, "Research and Implementation of the Localization Algorithm Based on RSSI Technology," Journal of Networks, vol. 9, pp. 3135-3142, 2014.

[3] P. Xie, J. H. Cui, and L. Lao, "VBF: vector-based forwarding protocol for underwater sensor networks," Networking Technologies, Services, and Protocols; Performance of Computer and Communication Networks; Mobile and Wireless Communications Systems, pp. 1216-1221, 2006.

[4] J. Guevara, A. Jiménez, J. Prieto, and F. Seco, "Auto-localization algorithm for local positioning systems," Ad Hoc Networks, vol. 10, pp. 1090-1100, 2012.

[5] I. Vasilescu, K. Kotay, D. Rus, M. Dunbabin, and P. Corke, "Data collection, storage, and retrieval with an underwater sensor network," in Proceedings of the 3rd international conference on Embedded networked sensor systems, pp. 154-165, 2005.

[6] A. Rahman, V. Muthukkumarasamy, and E. Sithirasenan, "Coordinates Determination of Submerged Sensors Using Cayley-Menger Determinant," in Distributed Computing in Sensor Systems (DCOSS), IEEE, pp. 466-471, 2013.
[7] A. Rahman, V. Muthukkumarasamy, and X. Wu, "Coordinates and Bearing of Submerged Sensors Using a Single Mobile Beacon (CSMB)", Journal of Networks, Vol. 10 No. 8, 2015.

[8] A. Rahman, V. Muthukkumarasamy, and E. Sithirasenan, "Localization of Submerged Sensors Using Radio and Acoustic Signals with Single Beacon," in Ad-hoc, Mobile, and Wireless Network. LNCS. vol. 7960, J. Cichoń, M. Gẹbala, and M. Klonowski, Eds., ed: Springer Berlin Heidelberg, pp. 293-304, 2013.

[9] V. Chandrasekhar, W. K. G. Seah, Y. S. Choo, and H. V. Ee, "Localization in underwater sensor networks: survey and challenges," in International Conference on Mobile Computing and Networking: Proceedings of the 1st ACM international workshop on Underwater networks,pp. 33-40, 2006.

[10] Z. Zhou, J. H. Cui, and S. Zhou, "Efficient localization for large-scale underwater sensor networks," Ad Hoc Networks, vol. 8, pp. 267-279, 2010.

[11] T. C. Austin, R. P. Stokey, and K. M. Sharp, "PARADIGM: a buoybased system for AUV navigation and tracking," in OCEANS 2000 MTS/IEEE Conference and Exhibition, pp. 935-938 vol.2, 2000.

[12] J. C. Prieto, A. R. Jiménez, J. Guevara, J. L. Ealo, F. Seco, J. O. Roa, and F. Ramos, "Performance evaluation of 3D-LOCUS advanced acoustic LPS," IEEE Transactions on Instrumentation and Measurement, vol. 58, pp. 2385-2395, 2009.

[13] P. Duff and H. Muller, "Autocalibration algorithm for ultrasonic location systems," in Seventh IEEE International Symposium on Wearable Computers, 2003. Proceedings, pp. 62-68, 2003.

[14] E. Olson, J. Leonard, and S. Teller, "Robust range-only beacon localization," in Autonomous Underwater Vehicles, IEEE/OES, pp. 6675, 2004.

[15] J. Guevara, A. R. Jimenez, A. S. Morse, J. Fang, J. C. Prieto, and F. Seco, "Auto-localization in Local Positioning Systems: A closed-form rangeonly solution," in IEEE International Symposium on Industrial Electronics (ISIE), pp. 2834-2840, 2010.

[16] A. Rahman and V. Muthukkumarasamy, , "Localization of Submerged Sensors with a Single Beacon for Non-Parallel Planes State", in 10th International conference on Ubiquitous and Future Networks (ICUFN), pp. 525-530, 2018. 